\title{
BMJ Open AGT-Reha-WK study: protocol for a non-inferiority trial comparing the efficacy and costs of home-based telerehabilitation for shoulder diseases with medical exercise therapy
}

\author{
Bianca Steiner (D) , ${ }^{1}$ Lena Elgert (D) , ${ }^{2}$ Reinhold Haux (D) , ${ }^{1}$ Klaus-Hendrik Wolf (D) ${ }^{2}$
}

To cite: Steiner B, Elgert L, Haux R, et al. AGT-Reha-WK study: protocol for a noninferiority trial comparing the efficacy and costs of homebased telerehabilitation for shoulder diseases with medical exercise therapy. BMJ Open 2020;10:e036881. doi:10.1136/ bmjopen-2020-036881

- Prepublication history and supplemental material for this paper are available online. To view these files, please visit the journal online (http://dx.doi. org/10.1136/bmjopen-2020036881).

Received 08 January 2020 Revised 10 June 2020 Accepted 14 August 2020

D) Check for updates

C Author(s) (or their employer(s)) 2020. Re-use permitted under CC BY-NC. No commercial re-use. See rights and permissions. Published by BMJ.

${ }^{1}$ Peter L. Reichertz Institute for Medical Informatics of TU Braunschweig and Hannover Medical School, Technische Universität Braunschweig, Braunschweig, Germany ${ }^{2}$ Peter L. Reichertz Institute for Medical Informatics of TU Braunschweig and Hannover Medical School, Hannover, Germany

Correspondence to

Bianca Steiner;

bianca.steiner@plri.de

\section{ABSTRACT}

Introduction Shoulder lesions rank among the top 15 diagnoses accounting for days of incapacity to work. Inpatient or full-day outpatient rehabilitation are some of the standard therapies. For sustainable rehabilitation, continuation of rehabilitation after discharge from a rehabilitation centre is vital. Besides medical exercise therapy (MET), home-based physical exercise programmes are used. To monitor exercise quantity and quality, AGTReha, a health-enabling technology for home rehabilitation, has been developed and evaluated in a pilot study for technical feasibility and acceptance. To integrate the digital therapeutic AGT-Reha into regular healthcare processes, an efficacy evaluation is required.

Methods and analysis AGT-Reha-WK is a prospective, monocentric, non-randomised, unblinded non-inferiority trial. Primary objective is to investigate whether AGT-Reha enhanced home-based exercise training is non-inferior to MET as standard aftercare. Secondary objective is to compare the costs of both therapies. Efficacy as medical success (primary outcome) is examined with regard to ability to work, return to work and sustainability of training (secondary outcomes). The outcome measure for non-inferiority is shoulder function (pain and disability) assessed by the standardised Shoulder Pain and Disability Index (SPADI). The non-inferiority margin is set to 10 points on SPADI score using a $95 \% \mathrm{Cl}$. Subjects will be recruited at the Rehabilitation Center Bad Pyrmont, Germany. The total number of subjects should be 84 (42 per group). Treatment takes 6 months per patient. Subjects will be assessed at four time points: pre-baseline (admission to rehabilitation centre), baseline (discharge from rehabilitation centre), post-therapy and follow-up (3 months post-therapy).

Ethics and dissemination Ethics approval was granted by the Ethics Committee of Hannover Medical School (ethics approval no: 7313). Results of the trial are planned to be published in a peer-reviewed journal.

Trial registration number German Clinical Trials Register DRKS00011596. Registered 2 June 2017. Recruitment started on 3 March 2017, and it is expected to continue until December 2020.

Protocol version V2.0, 23 May 2018, Amendment 01: improved risk analysis, clarification of exclusion criteria to increase reproducibility, additional documentation with
Strengths and limitations of this study

- This study contributes to bring digital therapeutics to patients' homes, an important additional 'institution' for healthcare.

- Implementation of an efficacy study at patients homes over a period of 6 months, including both an evaluation in clinical routine and patients' everyday life.

- Increased objectivity by assessing shoulder function as self-report and additional standardised measurement of range of motion using a goniometer.

- No randomisation due to clinical routine and clear preferences of patients.

- Simple integration of health economic evaluations as secondary outcome into the non-inferiority trial (piggyback design)

OpenClinica; these changes have no effect on structural equality.

\section{INTRODUCTION}

One promise of health-enabling technologies (HETs) is to improve quality and effectiveness of care by providing new digital therapeutics. ${ }^{1}$ While there is a breadth of newly developed HET, few demonstrate their efficacy in clinical trials. This might be due to the fact that there are only a few examples for studies that have been carefully adapted to the requirements to study the efficacy of HET. Most studies evaluating telerehabilitation systems reported in literature have some shortcomings. For instance, in most cases, the number of subjects is rather low with less than $15 .^{2-6}$ Another criticism is that subjects did not train for an extended period at their homes. ${ }^{2-46-10}$ Some studies are not controlled, ${ }^{3}{ }^{10}$ while others do not even include patients. ${ }^{2}$ Against this background, this publication introduces the study design to evaluate a HET 
called AGT-Reha which was developed to assist patients with chronic shoulder diseases during their home-based rehabilitation.

Musculoskeletal diseases are a leading cause of chronic pain, physical impairment and loss of quality of life. ${ }^{11}$ They often lead to absence from work, which causes high socioeconomic costs. ${ }^{12}$ Shoulder lesions as one example of musculoskeletal diseases ranks among the top 15 diagnoses accountable for the highest proportion of days of incapacity to work (19.31 days per 100 insurance years). ${ }^{13}$ In Germany, standard of care includes pain-reducing drugs, inpatient and full-day outpatient rehabilitative care. ${ }^{14}$ To sustain the effect of inpatient rehabilitation, a continuation after discharge is crucial. Medical exercise therapy (MET) is the most often prescribed measure. Complementary, the patients are advised to carry out self-reliant home-based exercise programmes. ${ }^{15}$ However, these physiotherapeutic exercises are often performed incorrectly or not as frequently as recommended. To improve exercise frequency and quality, the home rehabilitation system AGT-Reha was developed. It has shown its technical feasibility and user acceptance of HETbased telerehabilitation in a pilot trial. ${ }^{16}$ The aim of the described study in this publication is to show the efficacy of AGT-Reha compared with the current standard of care MET.

\section{Previous findings (pilot study)}

AGT-Reha-WK is part of the collaborative research project 'Health-enabling Technologies for Rehabilitation Training' (AGT-Reha). The aim of the project is to investigate the use of HETs for home-based telerehabilitation in the outpatient postrehabilitation phase. The project is structured as follows: (1) prototypical implementation and evaluation of feasibility of telerehabilitation training using HET, (2) empirical testing at patients' home through a feasibility study (AGT-Reha-P2) and (3) analysis of the efficacy through a non-inferiority trial (AGT-Reha-WK).

The prospective, single-arm, monocentric, explorative pilot study AGT-Reha-P2 showed the technical feasibility of AGT-Reha at patients' home. ${ }^{16}$ AGT-Reha enables patients to exercise independently at home, while the corresponding data on exercise quality and quantity are automatically forwarded via a web interface to physical therapists. AGT-Reha-P2 also shows the general acceptance by treating physical therapists and patients. ${ }^{16}$ All the 12 participants would recommend the system, 10 would reuse it and 7 would continue to use it after 3 months of therapy. Almost all participants perceive AGT-Reha as a motivational assistance for their home-based training. Secondary outcome of AGT-Reha-P2 was the medical rehabilitation success to estimate the expected effect size for aftercare with AGT-Reha in preparation of sample size calculation for AGT-Reha-WK. Therefore, the shoulder function (pain and disability) was assessed with the German version of the Shoulder Pain and Disability Index (SPADI) at discharge from rehabilitation centre and post-therapy. The difference in means (MD) in SPADI was -17 points with a SD of 15.53 .

\section{Objectives}

The primary objective of AGT-Reha-WK is to investigate whether home-based telerehabilitation with AGT-Reha is non-inferior to the standard aftercare, MET. Efficacy, as medical rehabilitation success, that is, shoulder function (primary outcome), is evaluated with respect to ability to work, return to work, as well as continuity and sustainability of training (secondary outcomes). Since there are already established aftercare measures in the outpatient postrehabilitation phase, AGT-Reha-WK comparatively investigates economic aspects of AGT-Reha (secondary objective).

The findings of AGT-Reha-WK will serve to facilitate a decision on the deployment and establishment of AGTReha as an alternative or supplement to other forms of aftercare.

\section{METHODS AND ANALYSIS \\ Study design}

AGT-Reha-WK is a prospective, phase III, non-inferiority trial using a parallel arm design. Single study centre is the Rehabilitation Center Bad Pyrmont. For logistical reasons, for example, delivery and pick-up of AGT-Reha systems, and the assessment procedures, randomisation and blinding are not possible (non-randomised, unblinded). Participation in the study is voluntary. In total, 84 subjects need to be included, 42 per group. After 3 weeks of inpatient rehabilitation at the Rehabilitation Center Bad Pyrmont, subjects in the intervention group undergo physical training at home with AGT-Reha, while subjects in the control group receive their prescribed MET, each for 6 months. This results in an active study duration of 7 months per subject with a follow-up after additional 3 months. In consideration of available AGTReha systems, the estimated handover times of systems, and the number of recruitable shoulder patients available at the Rehabilitation Center Bad Pyrmont, the estimated total study duration is 2.5 years.

\section{Patient population and eligibility criteria}

All subjects are working-age (18 to 65 years) patients of the participating rehabilitation centre currently undergoing inpatient rehabilitation for (chronic) shoulder pain as a result of shoulder complaints. This includes patients with 10th revision of the International Statistical Classification of Diseases and Related Health Problems (ICD-10) codes of the categories M19 'other arthrosis', especially of the shoulder joint, and M75 'shoulder lesions'. For treatment in both groups, it is particularly important that these patients can perform the selected exercises without risks of injuries. Therefore, patients are excluded who are not able to or should not perform these exercises. This also pertains in case of a new disease or an acute relapse during the study. A decision on this will be 


\section{Box 1 Inclusion and exclusion criteria}

\section{Inclusion criteria}

- Chronic shoulder pain due to shoulder complaints (10th revision of the International Statistical Classification of Diseases and Related Health Problems codes M19.1, M19.11, M19.21, M24.41, M75.0.-M75.9 (excluding M75.2, M75.6, M75.7).

- Age between 18 and 65 years.

- Sufficient German or English skills to follow simple instructions of AGT-Reha and to fill in questionnaires.

- Ability to perform the implemented exercises without any health risk.

- Place of residence in Lower Saxony.

- Pension insurance at DRV.

\section{Exclusion criteria}

- Missing declaration of consent.

- Participation in another clinical trial, either currently or within the last 30 days.

- Pregnancy and lactation.

- Other form of aftercare in parallel.

- Exercises cannot be or should not be performed.

- Physical or mental impairments which avoid proper executions of exercises.

- Body mass index $>35 \mathrm{~kg} / \mathrm{m}^{2}$.

- Limited willingness to use technology (only AGT-Reha group).

- No basic computer skills (only AGT-Reha group).

made by a treating physical therapist or physician based on a subjective appraisal of the patients' state of health and the individual therapeutic objectives. Furthermore, patients should not receive any other form of aftercare in parallel.

The AGT-Reha system has to be able to adequately capture and assess the patients' movements. Therefore, a body mass index of over $35 \mathrm{~kg} / \mathrm{m}^{2}$ is one exclusion criteria. To guarantee first-level support and personal examinations post-therapy at patients' home, subjects must reside in Lower Saxony and be pension insured with Deutsche Rentenversicherung Braunschweig-Hannover (DRV). All eligibility criteria are listed in box 1 .

\section{Interventions}

AGT-Reha-WK seeks to systematically compare the efficacy and costs of home-based telerehabilitation with AGTReha to MET. Thus, a parallel group design with a control group and an intervention group is planned.

\section{Medical exercise therapy}

MET is the quasi standard in Germany. Subjects in the control group receive their prescribed MET. In most cases, MET is performed over a period of 6 months. ${ }^{17}$ With a training frequency of twice a week, subjects exercise for $90 \mathrm{~min}$ in a certified facility of their choice, for example, outpatient rehabilitation centre or gym. As per guideline, training starts with a 10 to 15 min warm-up on two different training devices. Afterwards patients carry out five different exercises with three sets of 20 repetitions. The exercise plan should include both exercises for muscle strengthening and mobility ( $80 \%$ of exercises)

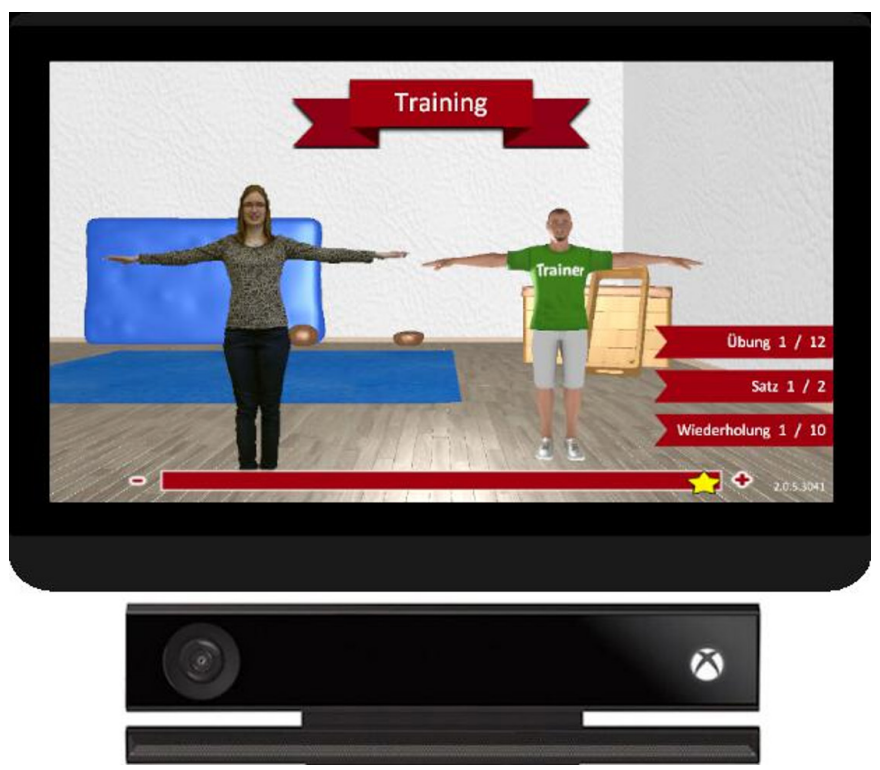

Figure 1 AGT-Reha training scene.

as well as cardiovascular training for overweight or obese patients. ${ }^{18}$ Patients are supervised by physical therapist. For each patient, an individual exercise plan (exercises, number of exercises, number of repetitions, difficulty levels) is compiled according to age, endurance and the patients' own assessment.

\section{Therapy with AGT-Reha}

Subjects in the intervention group perform AGT-Reha enhanced physical training at home for 6 months. The subjects should exercise regularly once on at least 5 days a week for a sustainable outcome. Exercise sessions last on average $30 \mathrm{~min}$.

AGT-Reha consists of an all-in-one personal computer (PC) and the marker-less motion capture system (depth camera) 'Kinect for Windows' (see figure 1). The selfdeveloped AGT-Reha client software guides patients through 10 physical exercises (see online supplemental additional file 1). These exercises have been specifically defined in cooperation with physical therapists of the Rehabilitation Center Bad Pyrmont for patients with (chronic) shoulder diseases. Treating physical therapists can individually adapt the training for each patient (exercises, number of repetitions, different difficulty levels for single exercises). For this purpose, they can access the training data (exercise quantity and quality) via web interface.

When the all-in-one PC is switched on, training with AGT-Reha starts automatically after a short calibration phase ensuring the correct positioning of patients in front of the depth camera. Following a short video explaining the upcoming exercise, AGT-Reha guides the patient through the first exercise. The training scene shows a three-dimensional visualisation (trainer avatar), showing the intended exercise execution. At the same time, the depth camera detects the patient's movements and mirrors the user on the screen. A coloured bar ranging 
from ' + ' to '-' on the bottom of the screen provides a continuous evaluation of exercise execution. This way, patients can easily detect and correct wrong movements. A pause of $30 \mathrm{~s}$ follows each exercise, as it is advised by physical therapists. During this time, a summary of execution quality-this is, accuracy, balance and speed-is displayed followed by the introduction of the next exercise.

\section{Risk analysis}

For patients of the AGT-Reha group, a comprehensive risk analysis was conducted in accordance with 'the basic rules of training' by Haber. ${ }^{19}$ The following aspects were examined in more detail to assess potential risks of injuries and overstress: quantification and appropriateness of training, selection of proper movement patterns, individualisation of training and information of patients. Standard frequency and intensity of training with AGT-Reha (five times a week, $30 \mathrm{~min}$ ) was specified together with physical therapists. Treating physical therapists are able to individually adjust the duration, intensity and frequency of the AGT-Reha training for their patients. It is possible to remove single exercises from sessions, change the number of repetitions and select the dumbbells' weights. Every decision is made telephonically in consultation with the patient. In addition, monthly phone calls are made by a study assistant. If a patient indicates training is too easy or too hard, contact with the treating physical therapist at the Rehabilitation Center Bad Pyrmont will be established.

\section{Outcome measures}

Subjects will be assessed at four time points: $\left(\mathrm{t}_{0}\right)$ prebaseline at admission to rehabilitation centre, $\left(\mathrm{t}_{1}\right)$ baseline at discharge from rehabilitation centre, $\left(\mathrm{t}_{2}\right.$, primary endpoint) post-therapy 6 months after $\mathrm{t}_{1}$, and $\left(\mathrm{t}_{3}\right)$ follow-up 3 months post-therapy (see table 1). Shoulder function will be used as primary outcome measure. As secondary outcome measures costs, shoulder mobility, changes in shoulder function over time, continuity and sustainability of training, as well as ability to work, subjective prognosis of work capacity and the work situation of patients will be assessed. All measurements at $\mathrm{t}_{0}$ and $\mathrm{t}_{1}$ are carried out in the rehabilitation centre. The measurements at $t_{2}$ and $t_{3}$ are done in a phone interview in which a study assistant jointly fills out all required questionnaires with a patient. Measurement of shoulder mobility is done on a separate personal appointment at subjects' home $\left(\mathrm{t}_{2}\right)$.

\section{Assessment of shoulder function}

The German version of the reliable and valid standardised questionnaire SPADI is used to assess the shoulder function. ${ }^{20}$ SPADI enables both recording of pain intensity by means of a Visual Analogue Scale and a questionnairebased survey of shoulder mobility (range of motion, ROM). The questionnaire consists of 13 items, 5 in the dimension pain and 8 in the dimension disability. ${ }^{21}$ For evaluation, the SPADI total score is used.
Table 1 Schedule of measures

\begin{tabular}{|c|c|c|}
\hline \multirow[b]{2}{*}{ Measures } & \multirow[b]{2}{*}{ Instruments } & Time point \\
\hline & & $\begin{array}{llll}t_{0} & t_{1} & t_{2}^{*} & t_{3}\end{array}$ \\
\hline \multicolumn{3}{|c|}{ Eligibility assessment } \\
\hline $\mathrm{BMI}$ & $\begin{array}{l}\text { Scale with built-in } \\
\text { stadiometer }\end{array}$ & $x$ \\
\hline $\begin{array}{l}\text { Technical } \\
\text { commitment }\end{array}$ & $\begin{array}{l}\text { Technology } \\
\text { commitment scale }\end{array}$ & $x$ \\
\hline $\begin{array}{l}\text { Technical } \\
\text { competence }\end{array}$ & $\begin{array}{l}\text { Self-developed } \\
\text { questionnaire }\end{array}$ & $x$ \\
\hline $\begin{array}{l}\text { Capturing of } \\
\text { movements by } \\
\text { AGT-Reha }\end{array}$ & Test training & $x$ \\
\hline
\end{tabular}

Primary outcome

\begin{tabular}{|c|c|c|c|c|}
\hline Shoulder function & SPADI & $x$ & $x$ & \\
\hline \multicolumn{5}{|l|}{ Secondary outcomes } \\
\hline Costs & $\begin{array}{l}\text { Self-developed } \\
\text { forms }\end{array}$ & $x$ & $x$ & \\
\hline $\begin{array}{l}\text { Shoulder mobility } \\
\text { (ROM) }\end{array}$ & Neutral-0-Method & $x$ & $x$ & \\
\hline $\begin{array}{l}\text { Continuity of } \\
\text { training }\end{array}$ & $\begin{array}{l}\text { Self-developed } \\
\text { questionnaire }\end{array}$ & & $x$ & \\
\hline $\begin{array}{l}\text { Sustainability of } \\
\text { training }\end{array}$ & $\begin{array}{l}\text { Self-developed } \\
\text { questionnaire }\end{array}$ & & & $x$ \\
\hline Ability to work & WAI & $x$ & $x$ & \\
\hline $\begin{array}{l}\text { Subjective } \\
\text { prognosis of work } \\
\text { capacity }\end{array}$ & SPE-Scale & $x$ & $x$ & \\
\hline Work situation & $\begin{array}{l}\text { Sociodemographic } \\
\text { core data set }\end{array}$ & $x$ & $x$ & \\
\hline
\end{tabular}

Tertiary outcomes

Pyschosocial health HEALTH-49

$\mathrm{x} x$

*Primary endpoint.

BMI, body mass index; HEALTH-49, hamburg modules for assessment of general aspects of psychosocial health for therapeutic practice; ROM, range of motion; SPADI, Shoulder Pain and Disability Index; SPE, Subjective Prognosis of gainful Employment; WAI, Work Ability Index.

\section{Assessment of costs}

Costs for an AGT-Reha system include following elements: (A) staff costs for therapeutic care of patients in the postrehabilitation phase, (B) staff costs for technical support, (C) maintenance and repair costs, (D) delivery costs and (E) procurement and replacement costs. Staff costs (A, B) are each documented on a separate form for physical therapists or technical staff. The time spent for calls with patients, use of the web interface, first-level support, as well as maintenance and repair has to be documented here continuously. Procurement and replacement costs (E) have to be estimated based on current prices for hardware and lifetime of all-in-one PCs, taking discounting into account. All other costs $(\mathrm{C}, \mathrm{D})$ have to be documented continuously during the study. 
Costs for MET are assessed via DRVs' settlement data. In addition to personal data, the period of aftercare, the first appointment and the number of treatments attended are stored.

\section{Assessment of shoulder mobility}

In addition to SPADI, ROM is measured using the Neutral0-Method ${ }^{22}$ by the same medical student, both in the rehabilitation centre $\left(t_{1}\right)$ and at patients' home $\left(t_{2}\right)$. The measurement is performed using a goniometer. ${ }^{23}$ The individual joint positions (relation to abduction/adduction, flexion/extension, external/internal rotation and external rotation/internal rotation at $90^{\circ}$ abduction) are directly documented in OpenClinica. ${ }^{24}$

\section{Assessment of continuity of training}

To assess the continuity of therapy, a self-developed questionnaire is used for answering questions about regularity and frequency of training sessions (see online supplemental additional file 2). In the AGT-Reha group, additional information on these aspects can be obtained directly from the data recorded by AGT-Reha. The questionnaire also records the causes for decrease in training frequency as well as activities performed in addition to the prescribed therapy, such as occupational therapy, gymnastics, fitness courses or swimming.

\section{Assessment of sustainability of training}

A self-developed questionnaire consisting of six questions is used to assess the sustainability of therapy (see online supplemental additional file 3). It asks to what extent the learned exercises or other forms of training are continued post-therapy. Here, in particular the frequency and reasons for a continued or discontinued training are recorded.

\section{Assessment of work-related aspects}

The work-related aspects are measured by different standardised questionnaires. The German short version of the Work Ability Index is used to assess ability to work. ${ }^{25}$ This is an easy-to-use, validated questionnaire covering seven dimensions with a total of 11 questions. ${ }^{25}$

To measure the subjective prognosis of work capacity, the Subjective Prognosis of gainful Employment (SPE scale) is used. ${ }^{26}$ This short three-item scale is implemented by certain pension insurers in Germany for sociomedical assessments. In the same way, the sociodemographic core data set is used to assess the work situation of patients.

\section{Allocation of patients}

On admission to the rehabilitation centre $\left(t_{0}\right)$, patients who meet the eligibility criteria are promptly allocated to one of the groups, either standard MET (control group) or home-based exercise training with AGT-Reha (intervention group). Clinical routine and patients' strong preferences make it difficult to randomise, but it is attempted. Therefore, patients are initially asked about their preferences. Patients who are willing to perform either of the two forms of aftercare are randomised. Randomisation is done by phone through a central randomisation facility. A randomisation list created by a random number generator allocates these patients to one of the two groups. Patients who strongly prefer one form of aftercare are assigned to the self-selected treatment group (explicit allocation). Once a patient is assigned, this assignment is entered into the patient's study record in OpenClinica.

Recruitment and allocation of patients has to be interrupted if no AGT-Reha system is available.

\section{Participants' timeline}

During their regular inpatient rehabilitation, all subjects undergo physical rehabilitation including learning and regularly performing the exercises used in AGT-Reha ( $\mathrm{t}_{0}-$ $\mathrm{t}_{1}$ ). Thereby, both groups receive individual and group therapies. All patients are asked to additionally perform the exercises on their own in their patients' room. For this purpose, subjects of the AGT-Reha group receive their AGT-Reha system immediately after group allocation. A detailed instruction for the training with AGTReha is given to every subject by their treating physical therapists. The day before discharge, all subjects have to fill out, jointly with a study assistant, the necessary questionnaires on shoulder function, work-related aspects and psychosocial health $\left(\mathrm{t}_{1}\right)$. ROM is be measured by the study assistant. Data are stored in the patient's study record and in OpenClinica.

During their 6-month home-based exercise training ( $\mathrm{t}_{1}$ $t_{2}$ ), subjects of the AGT-Reha group will exercise using AGT-Reha. For every training session, exercise data of patients are synchronised to make them available for physical therapists via the web interface. In case of abnormalities in training's frequency or quality, the treating physical therapist may contact the patient by phone to enquire about existing issues. On this basis, exercises can be adapted remotely via the web interface. Subjects of the control group take their MET in the prescribed frequency without further interventions $\left(\mathrm{t}_{1}-\mathrm{t}_{2}\right)$. Monthly phone interviews are conducted with all subjects to assess changes in shoulder function over time as well as training frequencies and course of the respective training.

Post-therapy $\left(t_{2}\right), 6$ months after $t_{1}$, the final assessment takes place. During a home visit, relevant questionnaires are filled out together with a study assistant. This study assistant (medical student) also carries out the ROM measurement and documentation. Three months later, a follow-up $\left(\mathrm{t}_{3}\right)$ is conducted by phone to record the sustainability of training.

For a detailed overview of participants' timeline, see figure 2.

\section{Sample size calculation}

Sample size calculation was carried out in cooperation with the Institute of Biometry of the Center for Biometry, Medical Informatics and Medical Technology of Hannover Medical School. For estimation of AGT-Reha's effect size, the results of the pilot study are used (MD $(\mathrm{SD})$ in SPADI $=-17(15.53))$. The effect size of MET is 


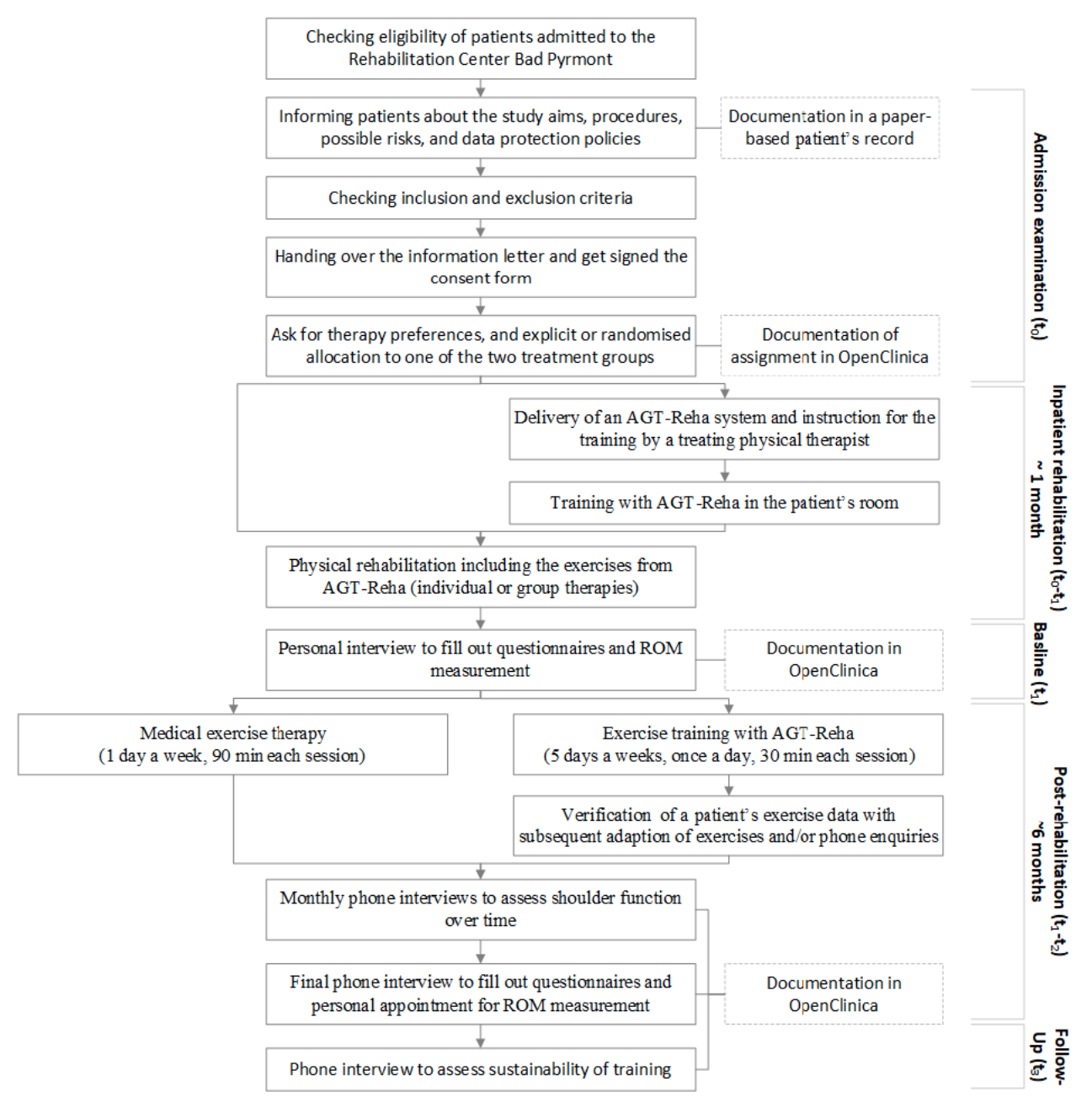

Figure 2 Participants' timeline (middle path=both groups; left path=control group; right path=intervention group). ROM, range of motion.

not known, neither from the pilot study nor from literature. However, it is assumed that the study leads to equal differences in means and SD in both groups. The noninferiority margin is selected on SPADI's test properties using literature references (conceptual design). Thus, a clinically relevant difference for SPADI is there with a change of $13^{27}$ or between 8 and $13^{28}$ points. Accordingly, the non-inferiority margin is set to -10 points $(\delta=-10)$. Assuming normal distribution, a one-sided two-sample t-test is planned with $\alpha=0.025$ and a given power of $80 \%$ (type II error=0.2). Consequently, 84 patients have to be included, 42 per treatment group. It is planned to observe drop-outs during the long observation period of 6 months and to continue recruiting, until a $10 \%$ over-recruitment (47 per group) is achieved.

\section{Statistical analysis}

Evaluation of non-inferiority

Primary objective of AGT-Reha-WK is to investigate whether the home-based exercise training with AGT-Reha is non-inferior to MET in terms of medical rehabilitation success measured by shoulder function. Therefore, differences in means between $t_{1}$ and $t_{2}$ in total SPADI score will be used $\left(\mathrm{H}_{0}: \mu_{\mathrm{MET}}-\mu_{\mathrm{AGT}} \leq-\delta ; \mathrm{H}_{1}: \mu_{\mathrm{MET}}-\mu_{\mathrm{AGT}} \geq-\delta\right)$.
Expecting no difference in variances of the treatment groups, evaluation uses an unpaired two-sample t-test for homogeneous variances. Neither a variable is known to be prognostic for therapeutic process, nor is a covariable needed. If the calculated $t$ value is greater than the critical $\mathrm{t}$ value, $\mathrm{H}_{0}$ will be rejected and $\mathrm{H}_{1}$ (significant difference between therapies) accepted. Afterwards, the principle of CI inclusion is used for the evaluation of non-inferiority. The confidence limit for the one-sided confidence level of $95 \%$ is calculated according to the applied t-test. The lower confidence limit $\left(\mathrm{C}_{0}\right)$ is then compared with the selected non-inferiority margin $(\delta=-10) . \mathrm{H}_{0}$ is rejected if $\mathrm{C}_{0}<\delta$. In this case, non-inferiority is proven.

Data are evaluated on the per-protocol principle. An intention-to-treat (ITT) analysis is additionally included to verify that the results do not depend on the underlying population.

\section{Cost comparison}

For the health economic evaluation, direct costs of MET and AGT-Reha are compared from the perspective of service providers. Two separate cost analyses applying an ITT analysis are used to calculate the average costs 
per patient. Costs of MET are evaluated using a topdown approach. This means, direct costs of MET in the observation period will be retrieved anonymously for each patient from the DRV, summed up and then averaged. Against this, costs of AGT-Reha are analysed by a bottom-up approach. Here, the individual elements of costs are added up. Procurement and replacements costs have to be calculated relatively on a patient based on the lifetime of an AGT-Reha system. Personnel costs for therapists and information technology (IT) staff must be determined according to the chosen business model for billing. The same applies to maintenance, repair and delivery costs. A subsequent summation and averaging of individual cost elements leads to a quantification of the average costs per patient.

\section{Secondary outcomes}

All secondary outcome measures are evaluated descriptively. This includes the calculation of median, mean, upper and lower quartiles, range and SD. A comparison will be done using box plots. For work-related aspects and ROM also an explorative evaluation is performed.

\section{Recruitment and consent}

Subjects will be recruited continuously at the Rehabilitation Center Bad Pyrmont, over a 2-year period. As part of the regular admission examination at the rehabilitation centre, eligible patients will be informed by a physician about the study aims, procedures, possible risks and data protection policies. Patients will also receive an information letter and a consent form (see online supplemental additional file 4). By signing this consent, patients agree to participate and to process their exercise data for improving AGT-Reha.

\section{Data management}

Data collection and documentation

During the study, both personal and medical data of subjects as well as data of physical therapists and IT staff are collected. Data recording is mostly paper-based. This relates especially to the data recorded at the rehabilitation centre $\left(t_{0}\right.$ and $\left.t_{1}\right)$ and to documentations of time required for therapeutic care of patients in the postrehabilitation phase by therapists and first-level support by IT staff. All paper-based documents must be archived in a patient's study record and then be transferred to OpenClinica for analysis. Exercise quality and quantity recorded by AGT-Reha will be temporarily stored in a file and then forwarded via a secure connection (HTTPS) to a server. Hence, this data can be accessed by physical therapist through the web interface. Camera images or depth data are neither transferred nor stored.

Adverse events are documented in a paper-based patient's study record, including time of event, intensity, countermeasures taken and the correlation to the therapy performed. If a patient discontinues the study (drop-out), the reasons are also recorded.

\section{Data monitoring and auditing}

At the end of a subject's study phase, all paper-based data are coded according to predefined coding instructions. Following, the corresponding codes, measured values and free texts are digitised in OpenClinica. Two study assistants independently encode the data to avoid coding, transmission and data acquisition errors. Complementary, a monitor ensures completeness of required documents and contributes to an early detection of problems during the study through regular visits to the participating rehabilitation centre.

\section{Data protection and security}

Data obtained during AGT-Reha-WK are used exclusively for data analysis and evaluation as part of the study. All patient-related and medical data recorded within AGTReha-WK are pseudonymously accessible for physical therapists and physicians of the Rehabilitation Center Bad Pyrmont and study assistants. Pseudonymisation is part of patient recruitment. A unique patient identification number (PIN) is given to each subject. Assignment of PIN takes place at the Rehabilitation Center Bad Pyrmont (central pseudonymisation). This enables physical therapists to deduce on individual patients, but not other study assistants (anonymisation). Only those study assistants conducting phone interviews or measuring ROM at patients' home also have access to the patients' names, addresses and phone numbers.

AGT-Reha does not interfere with the privacy of patients. Neither are insights into apartments possible nor are videos or sounds recorded. Training with AGT-Reha requires only a temporary use of video and depth images for real-time feedback of exercise quality. The AGT-Reha software performs the analysis locally. No transfer of raw data is required.

\section{Patient and public involvement}

Neither patients nor the public were involved in planning and design of this study. It is intended to disseminate the results to participating patients in an appropriate manner to be defined.

\section{Ethics and dissemination}

A written declaration of consent following the ethical principles of the Declaration of Helsinki was given by all subjects. All data protection and privacy aspects are designed according to the General Data Protection Regulation (European Union) 2016/679 and are approved by the data security officer of TU Braunschweig and the Ethics Committee of Hannover Medical School. Ethics approval for this non-inferiority trial was granted by the competent ethics committee. A steering committee for the projects and the study has been formed by involved parties. The steering committee is regularly informed about the ongoing study and meets at least twice a year. Further details on data protection and privacy can be requested from the authors. The results of AGT-Reha-WK are to be published in a peer-reviewed journal. 


\section{DISCUSSION}

Continuation of rehabilitation after full-day outpatient or inpatient rehabilitation is essential for sustainable rehabilitation success, especially for chronic diseases. HETsupported home-based rehabilitation as a digital therapy offers new possibilities for patients to continue their physical exercises with tele-therapeutic support. One telerehabilitation system supporting patients with shoulder diseases during their home-based aftercare is AGT-Reha. It guides patients through 10 physical exercises with automated real-time feedback and asynchronous feedback from physical therapists. To disseminate AGT-Reha in healthcare, an efficacy evaluation is crucial. Therefore, a phase III therapy study, called AGT-Reha-WK, in which the non-inferiority of AGT-Reha compared with MET will be assessed, was introduced with this study protocol.

Our study design has several limitations. One of the main objectives of AGT-Reha-WK is the health economic evaluation of AGT-Reha. However, the economic analysis was simply integrated into the non-inferiority trial (piggyback design) eclipsing the planning aspects of the cost analysis. For example, sample size calculation is based solely on statistical methods of non-inferiority trials. In general, health economic evaluations require a larger number of cases to create better heterogeneity among patients. ${ }^{29}$ A calculated sample size of $n=84$ presumes sufficient heterogeneity of patients for the health economic evaluation. Another issue of the piggyback design is a lack of explicit measurement points for determining direct costs for both therapies. Again, the focus is on the primary outcome, which may result in organisational difficulties in measuring the secondary parameters.

To prove the efficacy of a therapy, generally a comparison with a control group of patients who either remain untreated or receive a placebo or are treated with a therapy with recognised efficacy is necessary. ${ }^{30}$ In this study, MET, the standard aftercare, was selected as suitable comparative intervention, although this therapy was not proven to be effective in placebo-controlled clinical trials. Overall, there is little evidence-based research in the field of such interventions. ${ }^{31}$ This is due to the fact that therapies as these do not allow placebo-controlled studies. Also, a proof of efficacy against no treatment is excluded, since there are (officially) no untreated patients after discharge from rehabilitation centre. Therefore, the efficacy of MET is often assumed in similar studies. ${ }^{32-36}$ The limited evidence makes it difficult to estimate the actual effectiveness of MET for sample size calculation. Neither basic literature nor other publications provide information on the effect size. Overall, it is unclear whether the shoulder function is actually improved or whether only a deterioration is prevented through MET (or other forms of aftercare). According to medical guidelines and regulations, physical exercises contribute to maintain, restore and improve mobility, strength, endurance and coordination. ${ }^{37} 38$ The effects depend on the individual patient. Hence, for sample size calculation based on the results of the pilot study, a similar SD, measured with SPADI, in both groups is assumed.

There are also rather broad eligibility criteria for sample selection. In general, strict criteria should be defined to obtain a patient population with maximum delta. For instance, a stronger restriction of selected ICD-10 diagnoses would be conceivable. Since AGT-Reha is intended to be employed as an alternative or supplement to MET, which is used to treat all shoulder diseases described, this restriction does not appear to be suitable in practice.

Despite these limitations, with AGT-Reha-WK a clinical phase-III therapy study evaluating a digital therapy has been successfully designed. AGT-Reha-WK does not only feature a higher number of subjects compared with other studies but also represents clinical routine due to a therapy period of 6 months at patients' homes. Thereby, the study not only serves to evaluate the medical effectiveness of AGT-Reha but also the sustainability and continuity of training as a crucial element of rehabilitation services.

\section{Trial status}

Recruitment started in March 2017 and was expected to continue until April 2020. To date, 144 patients have been enrolled in the study $\left(\mathrm{n}_{\text {AGT-Reha }}=68, \mathrm{n}_{\text {MET }}=51\right)$. Twenty-five have been already excluded prior group allocation at the end of their clinical stay. Forty-three of them have fully completed the study (27 with AGT-Reha, 16 with MET). Due to the current COVID-19 pandemic, recruitment has been halted.

Acknowledgements The authors all persons involved in the AGT-Reha project for their assistance in planning AGT-Reha-WK, especially Birgit Saalfeld for constant discussions on technical aspects. Special thanks to the physical therapists of the Rehabilitation Center Bad Pyrmont for taking over the therapeutic support of patients during their inpatient rehabilitation and their home-based exercise programme with AGT-Reha.

Contributors BS was responsible for outlining the conception and design of the study protocol and drafting the manuscript. KHW substantially contributed to the overall design of the study and revised the manuscript. LE prepared major parts of the risk analysis for patients in the AGT-Reha group, coordinates the examinations and interviews with participating patients and revised the manuscript. RH provided statistical knowledge for the design of the underlying statistical model and reviewed the protocol. All authors read and approved the final manuscript.

Funding This work was supported by Deutsche Rentenversicherung Braunschweig-Hannover grant number 308.160 Leistung1-G.-Ref.RH. Open Access funding is provided by the German Research Foundation and the Open Access Publication Funds of the Technische Universität Braunschweig.

\section{Competing interests None declared.}

Patient and public involvement Patients and/or the public were not involved in the design, or conduct, or reporting, or dissemination plans of this research.

\section{Patient consent for publication Obtained.}

Provenance and peer review Not commissioned; externally peer reviewed.

Open access This is an open access article distributed in accordance with the Creative Commons Attribution Non Commercial (CC BY-NC 4.0) license, which permits others to distribute, remix, adapt, build upon this work non-commercially, and license their derivative works on different terms, provided the original work is properly cited, appropriate credit is given, any changes made indicated, and the use is non-commercial. See: http://creativecommons.org/licenses/by-nc/4.0/.

\section{ORCID iDs}

Bianca Steiner http://orcid.org/0000-0002-6762-8973

Lena Elgert http://orcid.org/0002-3394-4257

Reinhold Haux http://orcid.org/0001-5376-8660 
Klaus-Hendrik Wolf http://orcid.org/0000-0002-3806-462X

\section{REFERENCES}

1 Haux R, Koch S, Lovell NH, et al. Health-Enabling and ambient assistive technologies: past, present, future. Yearb Med Inform 2016;1:S76-91.

2 Chang CM, Chang YC, Hsiao BY, The design of a shoulder rehabilitation game system. International conference on Frontier computing - Theory, technologies and applications. Taichung, Taiwan. 4-6 Aug, 2010.

3 Da Gama AEF, Chaves TM, Figueiredo LS, et al. MirrARbilitation: a clinically-related gesture recognition interactive tool for an AR rehabilitation system. Comput Methods Programs Biomed 2016;135:105-14.

4 Dahl-Popolizio S, Loman J, Cordes CC. Comparing outcomes of kinect videogame-based occupational/physical therapy versus usual care. Games Health J 2014;3:157-61.

5 Macías-Hernández SI, Vásquez-Sotelo DS, Ferruzca-Navarro MV, et al. Proposal and evaluation of a telerehabilitation platform designed for patients with partial rotator cuff tears: a preliminary study. Ann Rehabil Med 2016;40:710-7.

6 Rizzo J-R, Thai P, Li EJ, et al. Structured Wii protocol for rehabilitation of shoulder impingement syndrome: a pilot study. Ann Phys Rehabil Med 2017;60:363-70.

7 Eriksson L, Lindström B, Ekenberg L. Patients' experiences of telerehabilitation at home after shoulder joint replacement. $J$ Telemed Telecare 2011;17:25-30.

8 Huang M, Lee S, Yeh S, et al. Intelligent frozen shoulder rehabilitation - a controlled study of rehabilitation at home after a shoulder joint operation. IEEE Intelligent Systems 2014;29:22-8.

9 Pekyavas NO, Ergun N. Comparison of virtual reality exergaming and home exercise programs in patients with subacromial impingement syndrome and scapular dyskinesis: short term effect. Acta Orthop Traumatol Turc 2017;51:238-42.

10 Tousignant $M$, Giguère A-M, Morin M, et al. In-home telerehabilitation for proximal humerus fractures: a pilot study. Int $J$ Telerehabil 2014;6:31-8.

11 Woolf AD, Erwin J, March L. The need to address the burden of musculoskeletal conditions. Best Pract Res Clin Rheumatol 2012;26:183-224.

12 Robert Koch-Institut. Gesundheit in Deutschland. Gesundheitsberichterstattung des Bundes. Gemeinsam getragen von RKI und Destatis. Berlin: H.Heenemann GmbH \& Co, 2015.

13 Grobe T, Steinmann S, aQua-Institut für angewandte Qualitätsförderung und Forschung im Gesundheitswesen $\mathrm{GmbH}$. Gesundheitsreport 2019 - Arbeitsunfähigkeiten. Hamburg: Techniker Krankenkasse, 2019.

14 Deutsche Rentenversicherung. Reha-Bericht 2018: die medizinische und berufliche rehabilitation Der Rentenversicherung Im Licht Der Statistik. Berlin: Deutsche Rentenversicherung Bund, 2018.

15 World Health Organization. Who guidelines on health-related rehabilitation (rehabilitation guidelines), 2014. Available: https:// www.who.int/disabilities/care/rehabilitation_guidelines_concept.pdf [Accessed 18 Oct 2019].

16 Steiner B, Elgert L, Saalfeld B, et al. Health-Enabling technologies for Telerehabilitation of the shoulder: a feasibility and user acceptance study. Methods Inf Med 2020;10. doi:10.1055/s-0040-1713685. [Epub ahead of print: 10 Aug 2020].

17 Deutsche Rentenversicherung. T-RENA - Trainingstherapeutische rehabilitationsnachsorge, 2020. Available: https://www.deutscherentenversicherung.de/DRV/DE/Reha/Reha-Nachsorge/T-RENA/ t-rena_node.html [Accessed 21 Apr 2020].
18 Diercks R, Bron C, Dorrestijn O, et al. Guideline for diagnosis and treatment of subacromial pain syndrome: a multidisciplinary review by the Dutch orthopaedic association. Acta Orthop 2014;85:314-22.

19 Haber P. Leitfaden Zur medizinischen Trainingsberatung rehabilitation bis Leistungssport. 4th edn. Berlin Heidelberg: Springer-Verlag, 2018.

20 Castro WHM, Jerosch J. Examination and diagnosis of musculoskeletal disorders: history - physical examination - imaging techniques - arthroscopy. New York: Thieme, 2011.

21 Breckenridge JD, McAuley JH, Pain S, et al. Shoulder pain and disability index (SPADI). J Physiother 2011;57:197.

22 Ryf C, Weymann A. The neutral zero method - a principle of measuring joint function. Injury 1995;26.

23 Celik D. Comparison of the outcomes of two different exercise programs on frozen shoulder. Acta Orthop Traumatol Turc 2010;44:285-92.

24 OpenClinica. OpenClinica reference guide, 2019. Available: https:// docs.openclinica.com [Accessed 10 Dec 2019].

25 Tuomi K, Ilmarinen J, Jahkola A, et al. Work ability index. Helsinki: Institute of Occupational Health, 1998.

26 Mittag O, Raspe $\mathrm{H}$. [A brief scale for measuring subjective prognosis of gainful employment: findings of a study of 4279 statutory pension insurees concerning reliability (Guttman scaling) and validity of the scale]. Rehabilitation 2003;42:169-74.

27 Roach KE, Budiman-Mak E, Songsiridej N, et al. Development of a shoulder pain and disability index. Arthritis Care Res 1991;4:143-9.

28 Roy J-S, MacDermid JC, Woodhouse LJ. Measuring shoulder function: a systematic review of four questionnaires. Arthritis Rheum 2009;61:623-32.

29 Schöffski O, Graf von der Schulenberg JM. Gesundheitsökonomische Evaluationen. Berlin, Heidelberg: Springer Verlag, 2012.

30 Schäfer H, Berger J, Biebler K-H, et al. GMDS Empfehlungen für die Erstellung von Studienprotokollen (Studienplänen) für klinische Studien. Informatik, Biometrie und Epidemiologie in Medizin und Biologie 1999;30:141-54

31 Huisstede BMA, Koes BW, Gebremariam L, et al. Current evidence for effectiveness of interventions to treat rotator cuff tears. Man Ther 2011;16:217-30.

32 Krischak G, Gebhard F, Reichel H, et al. A prospective randomized controlled trial comparing occupational therapy with home-based exercises in conservative treatment of rotator cuff tears. J Shoulder Elbow Surg 2013;22:1173-9.

33 Kromer TO, de Bie RA, Bastiaenen CHG. Physiotherapy in patients with clinical signs of shoulder impingement syndrome: a randomized controlled trial. J Rehabil Med 2013;45:488-97.

34 Østerås H, Torstensen TA, Østerås B. High-dosage medical exercise therapy in patients with long-term subacromial shoulder pain: a randomized controlled trial. Physiother Res Int 2010;15:232-42.

35 Ginn KA, Cohen ML. Exercise therapy for shoulder pain aimed at restoring neuromuscular control: a randomized comparative clinical trial. J Rehabil Med 2005;37:115-22.

36 Calis HT, Berberoglu N, Calis M. Are ultrasound, laser and exercise superior to each other in the treatment of subacromial impingement syndrome? A randomized clinical trial. Eur J Phys Rehabil Med 2001;47:375-80.

37 van der Meijden OA, Westgard P, Chandler Z, et al. Rehabilitation after arthroscopic rotator cuff repair: current concepts review and evidence-based guidelines. Int J Sports Phys Ther 2012;7:197-218.

38 Federal Joint Committee. Heilmittel-Richtlinien, 2019. Available: https://www.g-ba.de/richtlinien/12/ [Accessed 18 Dec 2019].

39 Neyer FJ, Felber J, Gebhardt C. Development and validation of a brief measure of technology commitment. Diagnostica2012;58:87-99. 